\title{
A Web Page for Automation Test Framework
}

\author{
Rucha Dave \\ U and P U Patel Department of Computer \\ Engineering, \\ Chandubhai S. Patel Institute of Technology, \\ Charotar University of Science and Technology, \\ CHARUSAT
}

\begin{abstract}
An Electric Vehicle Supply Equipment (EVSE) is the equipment which is used to charge Electric Vehicle (EV). This paper describes about how a web page can reduce the time and workload for testing the test scripts created for the complete system. Car simulator board is being used to simulate the action of the EV. It is not feasible to test number of test cases again and again as it is time consuming and increases manual work. Automation of the test cases is the main aim of this paper. To automate this test cases a web page is developed with the help of MEAN stack. It will also be helpful to automatically login to the mobile and web applications. An algorithm is explained for this complete process. It also explains about the implementation of the webpage. Modbus is being used to connect the program written in the system into the microchip. This does not imply that automation will completely replace manual testing, but will provide adequate support to the testing life cycle.
\end{abstract}

\section{Keywords}

Electric Vehicle Charging System (EVSE), Test automation, MEAN Stack, Test cases.

\section{INTRODUCTION}

An electric vehicle, additionally known as an energy unit, uses one or a lot of electric motors or transaction motors for propulsion. An electric car can be hopped through a system by electricity from off-vehicle sources, or could also be selfcontained with battery, star panels or an electrical generator to convert fuel to electricity.

EV charging station additionally known as Electric vehicle charging station, ECS (Electronic Charging Station) and EVSE (electric vehicle offer equipment), is a part in an infrastructure that provides electrical energy for the recharging of electrical vehicles, like plug-in electrical vehicles, together with electrical cars, neighborhood electrical vehicles and plug-in hybrids.

The electrical board which simulates the action of car is known as "Car Simulator Board". It contains different states which shows in which state the car simulator is presently.

Whenever the state of car simulator changes it reflects its values in all the other peripherals that are connected to it like mobile phone or web page. The process can be paused and released through different ways. It can be done by clicking the physical button which is attached to charging system. And it can also be done by using the mobile application or web application.

Here, the unique thing is the execution of the test cases are generated automatically. It is known as Test case Automation. For the test case automation web page is developed which will run the configuration file and creates the Configuration.Py file

\author{
Ritesh Patel, PhD \\ U and P U Patel Department of Computer \\ Engineering, \\ Chandubhai S. Patel Institute of Technology, \\ Charotar University of Science and Technology, \\ CHARUSAT
}

which contains all the configuration parameters which needs to apply for the automation purpose.

Here, in the system test cases are automated through the web page developed with the help of MEAN stack. We need to enter the unit type and configuration name in the webpage which is developed for automation purpose. Based on the unit type selected the other parameters are automatically updated. If we want change the configurations we can change it accordingly.

After insertion of the data once we save the configuration. We need to run the configuration, selection of test cases needs to be done. As we run the configuration file it automatically creates the Configuration.py file. After that it runs batch file which requires pytest framework. It stores the result in the database. It generates the report file which shows all the details about the process like errors, exception, results etc.

If anyone wants to run the saved configuration it can be directly done by "Saved Configurations" page.

We can even access the system remotely which is different apart from normal EVSEs. The remote access can be taken through webpages or through the Mobile phones. By entering the url of the website state of your Car Simulator can be known. Even the states of the car simulator can be changed by taking the remote access. It can also be accessed directly from the phone by using phone application.

Here, OCPP (Open Charge Point Protocol) is used for communication between Electric Vehicle Charging Stations and Central System. It is also known as a charging station network, similar to cell phones and cell phone networks.

OCPP is an application protocol - a language that allows communication between a compliant charge points, like the EV Link range, and any central network system.

\section{BACKGROUND}

The background aims to provide important information about the model that was used in this project. It also explains the different sorts of electric vehicles that are used in this study.

Electric vehicle are vehicles powered by electricity which means gasoline isn't used thereby reducing the pollution. Electric cars were first built in 19th century but its popularity took steep rise in early 2000s when prices of non-renewable sources started to rise. The popularity of electric vehicle can also be associated with the discovery of lithium ion battery which made cars lighter and also cheaper. Still it has very small number worldwide as it contributes just $2.5 \%$ of the total market share. This make electric cars isn't yet the main stream transportation device. However, it will surely be in the future. 
Battery Electric Vehicle: Battery electric vehicle uses electric motor and a battery which can be refiled by power grid. This process is achieved by connecting a cable between the vehicle and power source.

Plug in Hybrid Electric Vehicle, PHEV: Plug in hybrid electric vehicle uses electric engine as the primary source of prime mover and the combustion engine as secondary one. The major difference between PHEV and HEV is the ability of PHEV to charge the electric engine using power grid as compared to HEV which charges uses combustion. PHEV proves to be a better energy and resource efficient option then a conventional hybrid vehicle.

"An electric car's charger is on board the vehicle-buried within the car's guts to convert AC from your house to DC for charging the car's propulsion battery. Individuals still decision the wall-mounted box that provides 240 VAC to the automobile a "charger"”.

Charging station has four basic context, they are:

1. Residential charging station: This is typically used by connecting at home and has no user authentication on metering.

2. Charging where has parked: It a commercial partnership between house owners and organizations which will embrace parking station, mall parking and mini centers.

3. Charging at public charging station: This are basically used in extended journeys and charger can be set at next stop.

4. Battery swaps: It is helpful to fulfil the refueling expectation of the normal drivers.

Now will take a look on background for Test Case Automation Framework

1st Generation: Record and Playback

This type of scripting is known as Linear Scripting Framework. No writings of code to create function is required and addition to it the steps are in sequential order. The steps that the tester records are navigation, user input, or checkpoints and to conduct the test they plays the scripts back automatically. The best example is Selenium IDE.

It is impossible to maintain the large number of tests. Integration of these tests into a CI process then getting a proper reporting and configuring for different working environments is a big headache as it is a big challenge to automate more complex scenarios.

\section{2nd Generation: Modular and Data-Driven Framework}

- Modular-Based Testing Frameworks

The data was more complex and hard coded in record and playback scripts which were impossible to write. Because of this most of the vendors started to support to export the code as well as open-source tools through which any programming language exports recorded tests which can be edited and modified.

\section{- Data Driven Testing Frameworks}

Whereas, Data Driven Testing Frameworks help the user to test the test scripts and segregate the test script logic and its data. The external database helps to store the test data through segregation. The external databases can be XML files, Excel files, text files, CSV files, ODBC repositories, etc.
The main disadvantage is low code maintainability. Debugging and troubleshooting got harder because of datadriven frameworks. Apart from this the test's readability is worst because the test logic and test data are separated.

3rd Generation: Library and Keyword-Driven Frameworks

- Library Architecture Testing Framework

Module-Based Testing Frameworks builds the Library Architecture Testing Framework through fundamental and foundational process with some additional advantages. Here, segregation of application into common function or functions is done instead of dividing the application under test into test scripts.

- Keyword-Driven Testing Frameworks

"In a keyword-driven framework, each function of the application under test is laid out in a table with a series of instructions in consecutive order for each test that needs to be run". Similar to data-driven frameworks, the separation of test data and script logic in this framework, which is taken to a step further.

To keep the abstraction good which is getting harder and harder when there is slight modification in the workflow in some of the applications. To write more abstract code it requires engineers with in-depth programming knowledge to its best. As the abstractions gets higher, the tests also gets harder to read.

\section{4th Generation: BDD and Hybrid Frameworks}

- $\quad$ BDD Testing Frameworks

For developers, business analysts, testers etc. BehaviorDriven Development frameworks allows automation of functional validations which makes understanding and reading very easy. In this type of frameworks it is not necessary for the user to have a deep knowledge about the programming language. For this purpose different tools are available for BDD like Gauge, Cucumber, SpecFlow etc.

- Hybrid Testing Frameworks

As per the name it is understandable that it is a combination of all of the above frameworks. Here we get all kind of benefits which is related to different frameworks which makes this framework the best thing to do with.

This framework will give best test results so, it should be adapted easily. However, it also possesses many cons as it is a combination of all other frameworks, so based on other framework's cons Hybrid Testing framework also have some cons like maintainability and troubleshooting issues, only single platform of technology is supportable, not-so easy readable tests.

\section{5th Generation: Selenium-Based Frameworks}

\section{- Selenium-Based Testing Frameworks}

Till now, how to write the tests was less focused by all testing frameworks. Some people thought that it will be grateful to share the framework with the world and to open-source it which will make the framework "core". There are lots of frameworks which focuses on web automation and they are Atata Framework, Selenide, EPAM JDI, and Objectivity. Any type of abstraction can be added here as per the requirements like BDD or keywords.

The primary reason to build this type of framework is for web, which is helpful to get solution for API, desktop or mobile which needs to be created by yourself. This type of frameworks lacks in proper documentation. Whenever you 
have a problem in any case, just submit the issue for the complicated code. But the problem is that these technical guys develop there tools during their free period of time so we don't get any exact time frame on which they will reply. Even if they responds to your query we can't be sure that the issue is resolved or when it will get fixed is also known. Mostly these test frameworks makes tests more stable that too without adding new features by the only wrap WebDriver.

\section{5th Generation: Rise of AI, Full-Stack Frameworks}

- Full-Stack Test Automation Frameworks: True Future/Present

Some people takes this profession as a hobby, even some people take it as a side project which is not suitable. Professionally building and testing is required. Each and every test automation should be considered as a product only or else you are unable to rely and trust on it.

There should be no limits for the user for which platform they can use whether it is Linux, Windows, or Mac.

There are countless benefits for full-stack frameworks which makes these framework much better in lots of ways as compared to previous generation frameworks and tools. But to use these new generations' frameworks the QA engineers needs to have a good technical knowledge. They should be updated with the new and latest programming languages and should have good knowledge about high-quality code, architecture and design too.

Having the best generic framework which provides all of the above benefits still are not able to solve the current QA flaws and automation struggle which includes a "clean test environment, preparing the right test data, designing the right test, how to report the results in the best possible ways" and it goes on as it does not have a separate tool for reporting the issue which solves the issue is a liable way.

\section{CHALLENGES}

They say "automating chaos simply provides you quicker chaos," not solely faster, however (to paraphrase a song by kooky Punk) harder, faster, stronger... chaos. Automation will be associate degree impressive productivity booster within testing groups and a top quality foil for your systems once used properly. Here we will look at the most common test automation challenges.

\section{- $\quad$ Selecting and Using the Appropriate Tools}

Many groups don't get past this part because of many reasons. They will lack the experience to use a specific tool, the tool they require doesn't exist, the tool or set of tools don't supply a thousandth action coverage, the price of a tool exceeds the check budget, etc.

If a tool is out of the budget, do a fast price vs. profit analysis and gift your case. You'll be able to live the injury done by a previous bug you have got encountered and show what quantity time and cash you may have saved if you had the tool in place.

\section{- Identifying a Starting Strategy}

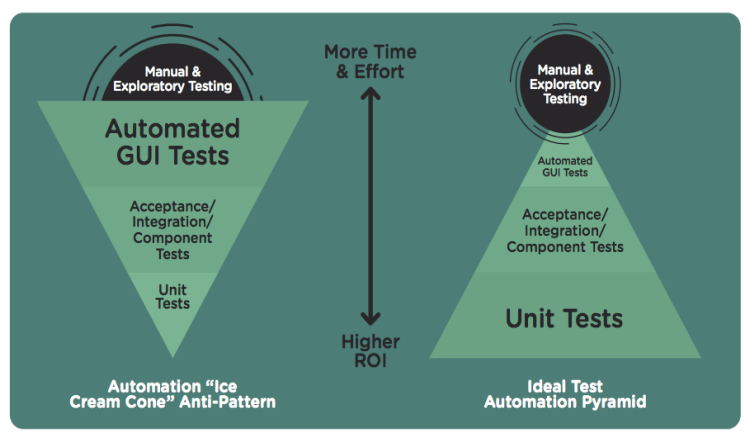

Fig 1: Difference between ideal automation and Ice cream Cone pattern

If we've all the tools and also the support to start automating, however what does one really alter and how? Unfortunately, the tools themselves don't tell you what to automate, even as new parents realize to their dismay that kids don't include a reference work. Can you raise a generation of outstanding machine-controlled tests or can they prove to be spoiled wrecks? In fact you'd hope for the former! Really, you can't alter everything therefore you have got to be strategic. You'll use 2 approaches to assist with this: risk-based testing and also the automation pyramid

Risk-based testing provides higher priority to testing the weather that are most in danger of failing that conjointly carry the best negative consequences if aforesaid failure happens.

The ice cream cone is good and tempting, however it might spoil your craving for automation! Following the ice cream cone approach can end in high levels of frustration as a result of it emphasizes automation on the UI level that employs additional brittle tests that break simply. Whereas, if you concentrate on automatic unit tests, you're serving to stop bugs or eliminate them rapidly as you undergo the software package development life cycle.

\section{PROPOSED MODEL / ALGORITHM}

\subsection{Algorithm}

The Above figure is the flow chart for web page development which will be used for test case automation. It explains the step wise process which will be executed once the full setup is ready. The following are the steps:

\section{Step 1. Creation of the configuration file.}

Open the webpage and enter the details and select the unit type that is required. Once the unit type is selected it will set all the parameters automatically.

Enter the username and password which is require to login to the web application and mobile phone application.

Step 2. After entering all the details we need to save the configuration. Which will redirect to the other page where all the configurations are saved. It will save the configuration into the database.

If anyone want to use previous configuration it will be available on this page. Just edit the selected configuration. It will fetch the data from the database.

After editing the configuration, save it. It will save the configured data into database. It will redirect to previous page.

Step 3. Here, now to run the configuration click on Run. It will direct to the page which contains all the evse features for 
which test cases are generated. Select the feature and number of iterations that needs to be run.

Step 4. As the selection of feature is done click on "Start Test" button. It will automatically save the configuration into the database with unique configuration id. It will start the test in the command prompt.

Step 5. It will create "Configuration.py" file which contains all the values of the parameters which is entered while creating the configuration. Every time when the configuration is started the configuration.py will get updated. It will automatically run the batch file under the py test framework.

Step 6. Now it will start the Pytest of that configuration.py file. It will work as per the testcase that has been selected. It will automatically get connected to the selected mobile phone which is selected during the configuration. It will also automatically login to the website which is used for charging. It will store all the updated data into database. It will insert the report data which will be saved to the database with configuration name. It will run the batch file with the Pytest framework.

Step 7.It will generate the report file which shows all the details of the process that have been executed using test cases and batch file.
This is how the whole process works for test cases automation. This brings us to the end of the one configuration process.

\subsection{Implementation Details (Tools and Technologies)}

A. Visual Studio Code: It is editor used to develop code for NodeJs as well as AngularJs. It is a lightweight but powerful source code editor which runs on your desktop and is available for Windows, MAC OS and Linux. It comes with built-in setup for JavaScript, Typescript and Node.js.

B. Mongo DB: It is a schema less NoSQL database system. Is saves data in binary JSON format which makes it easier to pass data between client and server? The concept is for collection and documentation. MongoDB provides high performance, high handiness, and easy scalability as a result of a cross-platform, document-oriented database.

C. Express JS: Express is a framework used for node.js development. It is a minimal web server built on node that provides all the essential functionality required for delivering web applications to the browser and mobile devices. It also allows you to handle routes, server and I/O stuff. It provides a robust features for web and mobile applications.

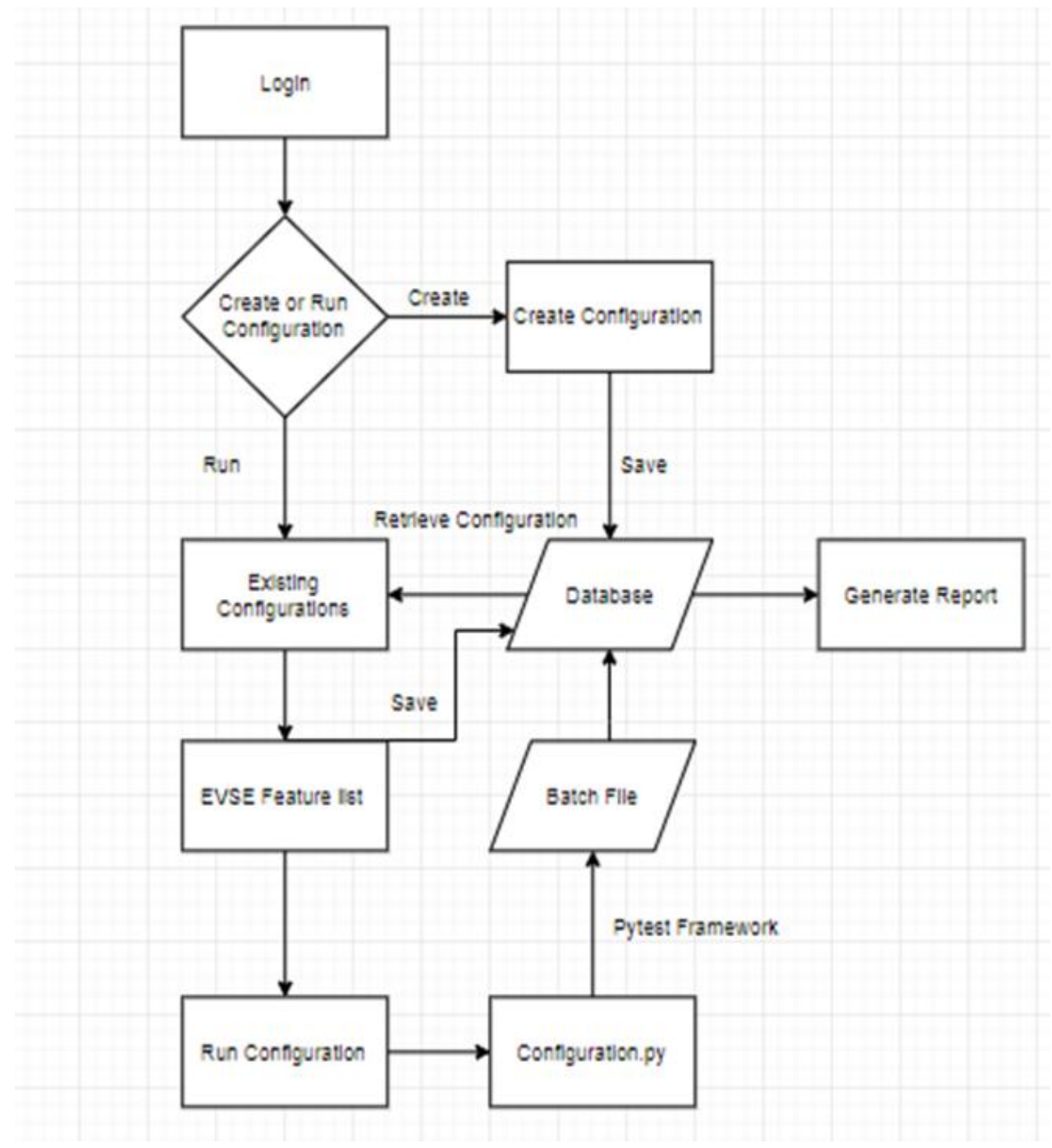

Fig 2: Algorithm 
D. Angular JS: It is a JavaScript framework developed by Google, used for the front end development and also provides two way data binding. It extends HTML attributes with Directives, and binds knowledge to HTML with Expressions. It eliminates much of unwanted code with its feature of data binding and dependency injection.

E. Node JS: It is a server side JavaScript execution environment used for developing server-side and networking applications. Node js efficient as it is lightweight, event driven and on-blocking I/O model. It has the most important scheme of open supply libraries within the world referred to as npm. It can even run JavaScript applications outside the browser.

F. SVN: SVN is a version control system, which creates a central place for all the developers. Here, all the developer's code is stored. It provides different trunks to keep separate working repositories.

G. Virtual Machine: A Virtual Machine (VM) is an emulation of a Physical Computer. Virtual machines are supported on computer architectures and supply functionality of a physical computer. Their implementations involves specialized hardware, software, or both

\subsection{Sequence Diagram of Test Case Automation Process}

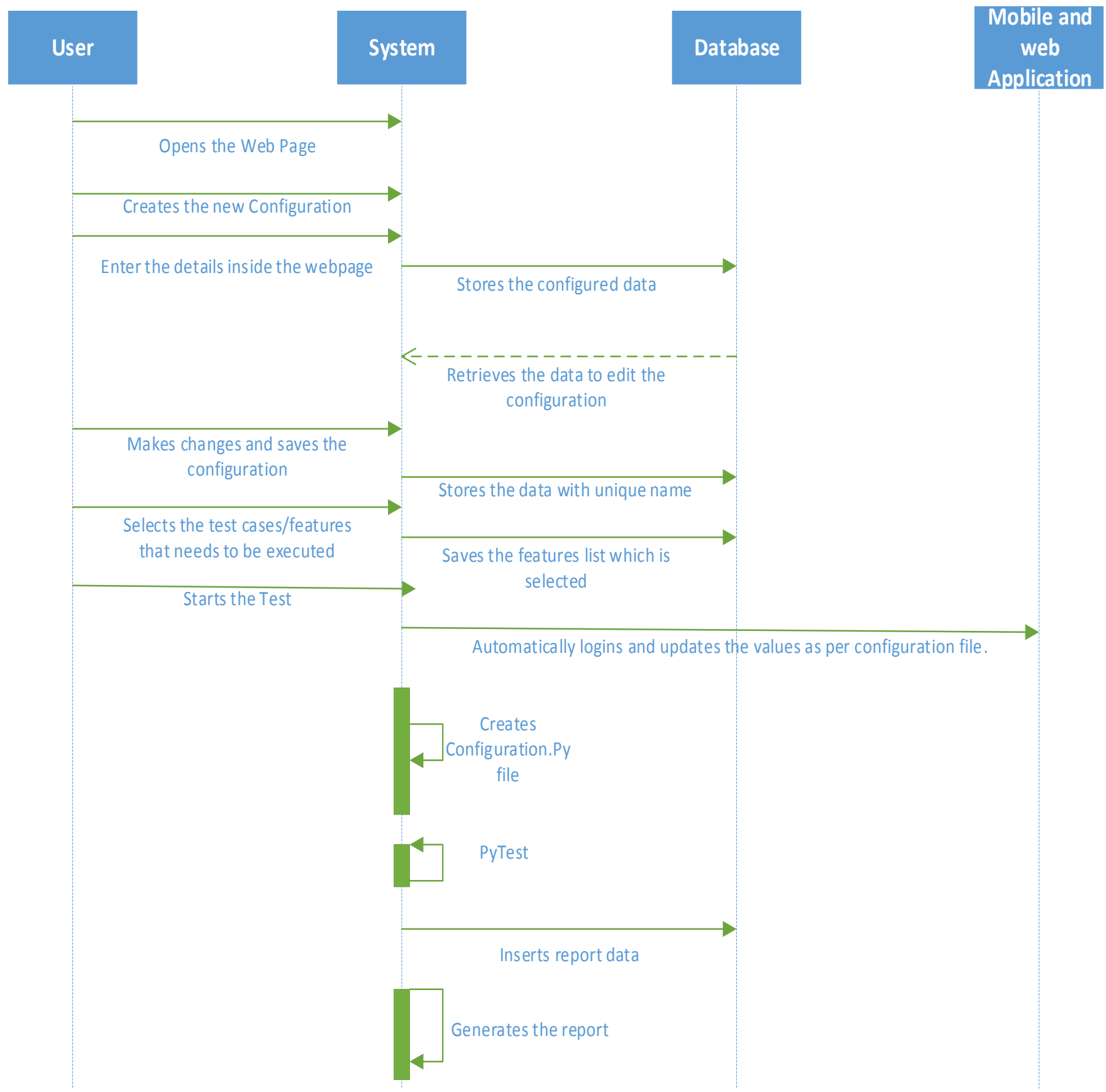

Fig 3: Sequence Diagram 
Above shown figure is the sequence diagram of the complete system which gives the idea about the implementation of the system.

\section{CONCLUSION AND FUTURE WORK}

Developing electric car is important in reducing greenhouse gas (GHG) emissions American vehicles are responsible for almost half of the GHGs emitted by vehicles globally, but make up 30 percent of cars in use, according to a 2006 report by Environment Defense. Electric vehicles will bring about new ways of refueling", a 2010 Ford press release stated. "Instead of topping off the tank with gasoline, customers will plug in". Here the brief description about EVSE is explained through different ways parameters. The solution for manual testing i.e Test case automation is done through web page creation. In future test automation will increase drastically. This makes finding a good charging setup important.

Here the webpage was created to automate the testing process. Now going forward we can automate the test scripts creation by its own knowledge with the help of some framework.

\section{REFERENCES}

[1] Bohn, Theodore, and Hal Glenn. "A real world technology testbed for electric vehicle smart charging systems and PEV-EVSE interoperability evaluation." 2016 IEEE Energy Conversion Congress and Exposition (ECCE). IEEE, 2016.

[2] Enyedi, Szilárd. "Electric cars-Challenges and trends." 2018 IEEE International Conference on Automation, Quality and Testing, Robotics (AQTR). IEEE, 2018.

[3] Nagar, Ishan, M. Rajesh, and P. V. Manitha. "A low cost energy usage recording and billing system for electric vehicle." 2017 International Conference on Inventive Communication and Computational Technologies (ICICCT). IEEE, 2017.

[4] Bohn, Theodore, Carlos Cortes, and Hal Glenn. "Local automatic load control for electric vehicle smart charging systems extensible via OCPP using compact submeters." 2017 IEEE Transportation Electrification Conference and Expo (ITEC). IEEE, 2017.

[5] Rubio, Juan E., Cristina Alcaraz, and Javier Lopez. "Addressing Security in OCPP: Protection Against Manin-the-Middle Attacks." 2018 9th IFIP International Conference on New Technologies, Mobility and Security (NTMS). IEEE, 2018.
[6] Nagowah, Leckraj, and Kreshnah Kora-Ramiah. "Automated complete test case coverage for web based applications." 2017 International Conference on Infocom Technologies and Unmanned Systems (Trends and Future Directions)(ICTUS). IEEE, 2017.

[7] Klinachev, N. V., and P. O. Shaburov. "Technique for debugging of the data exchange between the PC and microprocessor-controlled electromechanical systems based on the modbus RTU protocol." 2017 International Conference on Industrial Engineering, Applications and Manufacturing (ICIEAM). IEEE, 2017.

[8] Thummalapenta, Suresh, et al. "Automating test automation." Proceedings of the 34th International Conference on Software Engineering. IEEE Press, 2012.

[9] Torsel, Arne-Michael. "Automated test case generation for web applications from a domain specific model." 2011 IEEE 35th Annual Computer Software and Applications Conference Workshops. IEEE, 2011.

[10] Lehfuß, Felix, et al. "Reference architecture for interoperability testing of Electric Vehicle charging." 2015 International Symposium on Smart Electric Distribution Systems and Technologies (EDST). IEEE, 2015.

[11] Peng, Daogang, et al. "Design and development of Modbus/RTU master monitoring system based on embedded PowerPC platform." 2009 IEEE International Symposium on Industrial Electronics. IEEE, 2009.

[12] Klinachev, N. V., and P. O. Shaburov. "Technique for debugging of the data exchange between the PC and microprocessor-controlled electromechanical systems based on the modbus RTU protocol." 2017 International Conference on Industrial Engineering, Applications and Manufacturing (ICIEAM). IEEE, 2017.

[13] "Backend Frameworks | Pvtraining.Frameworks.Ca Easy Counter". Keywordniches.Com, 2019, http://www.keywordniches.com/search/backendframeworks.

[14] "Difference Between Ideal Automation And Ice Cream Cone Pattern - Google Search". Google.Com, 2019, https://www.google.com/search?q=Difference+between+ ideal+automation+and+Ice+cream+Cone+pattern\&sourc $\mathrm{e}=\operatorname{lnms} \&$ tbm $=$ isch $\&$ sa $=X \& v e d=0 a h U K E w i z 1 \mathrm{MfDwYzi}$ AhUPb30KHYXwAdoQ_AUIDygC\&biw=1366\&bih=6 25\#imgrc $=$ YD12kXY8jAntmM: 